\title{
Multi-material specific, targeted self-assembly of nanocrystal emitters using genetically engineered peptides on optoelectronic microchips
}

\author{
Gulis Zengin, ${ }^{1}$ Urartu Ozgur Safak Seker, ${ }^{2}$ Asli Koc, ${ }^{1}$ Evren Mutlugun, ${ }^{1}$ Ozgun Akyuz, ${ }^{1}$ Emre Sari, ${ }^{1}$ \\ Mehmet Sarikaya, ${ }^{3}$ Candan Tamerler, ${ }^{2,3}$ and Hilmi Volkan Demir ${ }^{1}$ \\ ${ }^{I}$ Department of Electrical and Electronics Engineering, Department of Physics, Nanotechnology Research Center, Institute of \\ Materials Science and Nanotechnology, Bilkent University, Ankara 06800, Turkey \\ ${ }^{2}$ Molecular Biology and Genetics, Istanbul Technical University, Maslak 34469, Istanbul, Turkey \\ ${ }^{3}$ Genetically Engineered Materials Science and Engineering Center, Materials Science and Engineering, University of \\ Washington, Seattle, Washington 98105, USA
}

Semiconductor nanocrystal quantum dots are exploited for use in a number of optoelectronic devices that typically consist of multiple materials [1,2]. However, these nanocrystal emitters are typically non-specifically assembled without recognizing surface materials in these devices. Non-specific assembly of quantum dots has been commonly realized by immobilizing quantum dots in a host polymer or closely packing them without using any host polymer. Such non-specific assembly undesirably leads to several drawbacks in their use on optoelectronic devices including applicability problems and main challenges in fabrication processes. Furthermore, specific assembly of nanocrystal emitters onto targeted regions in an optoelectronic device is required to make molecular scale devices. To address these demands, we demonstrated directed immobilization of the nanocrystal emitters only on the targeted specific regions homogeneously on multi-material patterned optoelectronic microchips in a controlled manner by using genetically engineered peptides for inorganics (GEPIs) as smart molecular linkers.

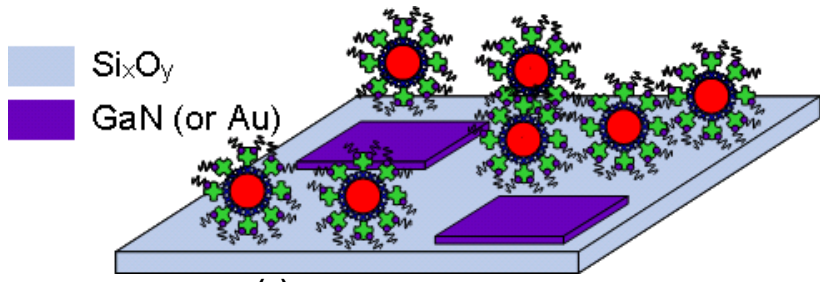

(a)

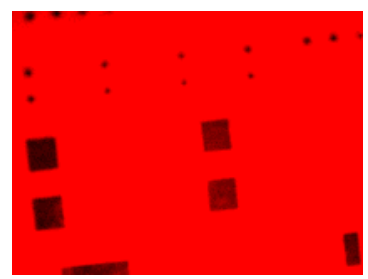

(b)

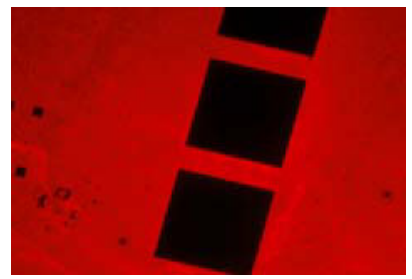

(c)

Fig.1 Multi-cross-specific binding of nanocrystals on silica surfaces using GEPIs: (a) schematic of red emitting SA-NC integrated with bio-QBP1 specifically binding on $\mathrm{Si}_{\mathrm{x}} \mathrm{O}_{\mathrm{y}}$ (but not on $\mathrm{GaN}$ or $\mathrm{Au}$ surfaces), and experimental demonstrations of specific binding of bio-QBP1+SA-NC assembly on silica in the presence of (b) GaN surfaces and (c) Au patterns on multi-material patterned optoelectronic devices.

In our study, we first examined the conventional method in which peptides and quantum dots were immobilized in film layer by layer, one after the other. For that, we used biotinylated GEPIs as nanocrystal linkers and streptavidin functionalized $\mathrm{CdSe} / \mathrm{ZnS}$ core-shell nanocrystals (SA-NCs) as emitters. Here GEPIs were selected and screened by cell surface or phage display methods and demonstrated to have strong affinity towards targeted materials [3-5]. Different from the commercial linkers, such as thiol or silane, these GEPIs feature specific material binding capability. In this study, we exploited in particular quartz binding peptide (QBP1) designed using a novel bioinformatics knowledge-based approach [6]. These samples were evaluated against a negative control group in which SA-NCs were immobilized in the absence of bio-QBP1. Even though the samples prepared with bio-QBP1 showed higher photoluminescence (PL) compared to the control group, they suffered from nonuniform distribution of the nanocrystal emitters on the silica surface and, consequently, their optical properties were not reproducible. These results indicated that the assembly of quantum dots in this case was diffusion limited due to stationary biotin film on the peptide functionalized quartz surface. This limited diffusion of quantum dots hindered the interaction between SA-NCs and bio-QBP1. 
To address this issue, in a new approach, we proposed decoration of SA-NC with bio-QBP1 before assembling them on the surface, by first mixing them in a buffer solution and then immobilizing them together on the surface (Figure 1). The samples prepared by the new method exhibited remarkable improvements in the photoluminescence intensity, with more uniform distribution of nanocrystal emitters on the surface (Figure 2). The samples prepared using the new method showed 270-fold enhancement in the photoluminescence intensity compared to the control group, while the samples in the conventional method had only 5 -fold enhancement.

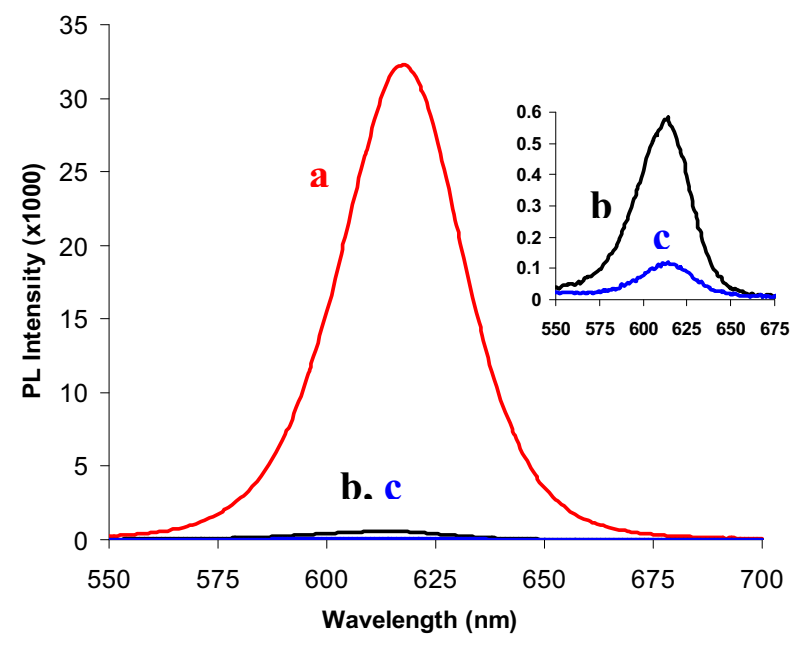

Fig.2 Photoluminescence (PL) of (a) the nanocrystal emitters prepared by our new method, (b) those prepared by the conventional method, and (c) the negative control group. The new method led to 270 times stronger PL than the control group and 55 times stronger PL than the conventional method; on the other hand, the conventional method led to only 4.9 times stronger PL than the control group.

In terms of binding properties, Figure 2 demonstrates that our new method was about 55 times better than the conventional one. In our studies, we also comprehensively explored the selective and specific self-assembly of the quantum dot emitters on multi-material patterned optoelectronic microchips consisting of minimum three distinct materials, e.g., Au/silica and GaN/silica (as shown in Figure 1). Here, by using our new method, we observed that QBP1 functionalized quantum dots differentiates the silica surfaces from other surfaces and bind only to the silica patterns on a multi-material microchip e.g., with Au or GaN (Figure 1(b) and 1(c)). In both cases, QBP1 covered nanocrystals showed significantly weak affinity towards $\mathrm{Au}$ and GaN surface when compared to the silica surfaces. In these characterizations, photoluminescence intensity of the silica patterns covered with red-emitting $\mathrm{NCs}$ was 9 times stronger than both $\mathrm{Au}$ and GaN surfaces, demonstrating multi-cross-specific binding on optoelectronic microchips for the first time.

In summary, we demonstrated material-specific binding of the quantum dot emitters hybridized with GEPI on multi-material patterned microchips. These proof-of-concept results open up new opportunities in nanophotonics, allowing for more specific and controlled assembly of quantum dots in optoelectronic devices and building of novel molecular organic-inorganic hybrid devices.

This work was supported in part by European Science Foundation EURYI Program, EU-PHOREMOST NoE511616, Marie Curie IRG MOON 021391, TUBA-GEBIP, and TUBITAK EEEAG 106E020, 104E114, 107E088, 107E297, 105E065, and 105E066 (HVD). This work was also supported in part by grants from US-NSF though the Genetically Engineered Materials Science Engineering Center (GEMSEC), and MRSEC (MS), and Turkish State Planning Organization via Advanced Technologies in Engineering (CT).

[1] Giepmans, B. N. G; Adams S. R.; Ellisman, M. H.; Tsien, R. Y. Science, 312 (5771), 217-224 (2006).

[2] Nizamoglu, S.; Zengin, G.; Demir, H. V. Applied Physics Letters, 92, 031102 (2008).

[3] Sarikaya, M; Tamerler, C.; Jen, A.K.Y.; Shulten K.; Baneyx, F. Nature Materials, 2 (9), 577-585 (2003).

[4] Whaley, S. R.; English, D. S.; Hu, E. L.; Barbara, P. F.; Belcher, A. M. Nature, 405, 665-668 (2000).

[5] Sano, K. I.; Sasaki, H.; Shiba, K. Langmuir, 21, 3090-3095 (2005).

[6] Oren E. E. et al.; Bioinformatics, 23, 2816-2822 (2007). 